\title{
Caracterización de Harina de Naranja (Citrus $x$ sinensis) para Uso Alimentario
}

\author{
Ericka Cristel Guerra Baños,
}

Egresada de Ingeniería en Alimentos, División Académica

Multidisciplinaria de los Ríos, Universidad Juárez Autónoma de Tabasco,

México

Martha Isela Baños Dorantes, Colegio de Bachilleres de Tabasco,Tabasco, México

Ana Laura Luna-Jiménez, Nicolás González-Cortés, Román Jiménez-Vera,

Cuerpo Académico Desarrollo Sustentable. División Académica Multidisciplinaria de los Ríos, Universidad Juárez Autónoma de Tabasco, México

\section{Resumen}

Una propuesta para disminuir la contaminación ambiental generada por los subproductos de la industria agroalimentaria es evaluar dichos desechos como aditivos para la industria alimentaria. El objetivo de este trabajo fue caracterizar harina de subproductos de la extracción de jugo de naranja dulce (Citrus $x$ sinensis) con la finalidad de emplearla como ingrediente alimentario. La caracterización fisicoquímica, funcional y microbiológica mostró que la harina posee una humedad adecuada para su almacenamiento $(8.73 \pm 0.11 \%)$ y una concentración media de proteínas (5.63 $\pm 0.14 \%$ ) para su empleo en la formulación de alimentos; el mayor componente fue el extracto libre de nitrógeno $(79.18 \pm 0.22 \%)$ y el menor, al extracto etéreo $(0.59 \pm 0.08 \%)$. Esta harina es una buena fuente de fibra ya que posee una concentración de fibra dietética total de $39.52 \pm 2.08 \%$, con un índice en la relación de fibra soluble e insoluble de 0.95 . A temperatura ambiente, se observó una capacidad de absorción de agua de $5.72 \pm 0.12 \mathrm{~g}$ de agua/g de harina, mientras que a temperaturas entre $60-90{ }^{\circ} \mathrm{C}$ se incrementó a $11.08 \pm 1.04 \mathrm{~g}$ de agua/g de harina. La capacidad de absorción de aceite fue de $3.95 \pm 0.07 \mathrm{~g}$ de agua/g de harina, a temperatura ambiente. Los mesófilos ( $\log 4.62 \pm 0.28 \mathrm{UFC} / \mathrm{g}$ ) se mantuvieron dentro de los valores máximos permitidos por la norma para harina de trigo. El empleo de la harina de subproductos de naranja como aditivo en alimentación humana apoyará para 
el diseño de nuevos productos alimentarios, disminuir la contaminación ambiental y agregar valor a la cadena productiva de cítricos.

Palabras clave: Harina de Naranja, Fibra Dietética, Subproductos Vegetales, Alimento Funcional

\title{
Characterization of Orange Flour (Citrus $x$ sinensis) for Food Use
}

\section{Ericka Cristel Guerra Banos,}

Egresada de Ingeniería en Alimentos, División Académica

Multidisciplinaria de los Ríos, Universidad Juárez Autónoma de Tabasco, México

Martha Isela Banos Dorantes, Colegio de Bachilleres de Tabasco,Tabasco, México

\section{Ana Laura Luna-Jimenez, Nicolás Gonzalez-Cortes, Román Jimenez-Vera,}

Cuerpo Académico Desarrollo Sustentable. División Académica Multidisciplinaria de los Ríos, Universidad Juárez Autónoma de Tabasco, México

\begin{abstract}
A proposal to reduce environmental pollution generated by the byproducts of the agri-food industry is to evaluate these wastes as additives for the food industry. The objective of this work was to characterize flour byproducts from the extraction of sweet orange juice (Citrus $\mathrm{x}$ sinensis) in order to use it as a food ingredient. The physicochemical, functional and microbiological characterization showed that the flour has adequate moisture for storage $(8.73 \pm 0.11 \%)$ and an average protein concentration $(5.63 \pm$ $0.14 \%$ ) for use in food formulation; the largest component was the nitrogenfree extract $(79.18 \pm 0.22 \%)$ and the minor, the ethereal extract $(0.59 \pm 0.08 \%)$. This flour is a good source of fiber since it has a total dietary fiber concentration of $39.52 \pm 2.08 \%$, with an index in the ratio of soluble and insoluble fiber of 0.95 . At room temperature, a water absorption capacity of $5.72 \pm 0.12 \mathrm{~g}$ of water / $\mathrm{g}$ of flour was observed, while at temperatures between $60-90^{\circ} \mathrm{C}$ it was increased to $11.08 \pm 1.04 \mathrm{~g}$ of water / $\mathrm{g}$ of flour. The oil
\end{abstract}


absorption capacity was $3.95 \pm 0.07 \mathrm{~g}$ of water / $\mathrm{g}$ of flour, at room temperature. The mesophilic ( $\log 4.62 \pm 0.28 \mathrm{CFU} / \mathrm{g}$ ) were kept within the maximum values allowed by the wheat flour standard. The use of the flour of orange by-products as an additive in human food will support the design of new food products, reduce environmental pollution and add value to the citrus production chain.

Keywords: Orange Flour, Dietary Fiber, Vegetable By-Products, Functional Food

\section{Introducción}

En la agroindustria, el procesamiento de la materia prima para su transformación genera una alta concentración de residuos, convirtiéndose en una de las principales problemáticas ambientales de la industria alimentaria. En algunos casos estos residuos son tratados para reducir el impacto negativo en el medio ambiente; otra solución es convertirlos en productos útiles y de mayor valor agregado para disminuir la problemática ambiental y generar ingresos económicos adicionales (Cury, Aguas, Martinez, Olivero \& Chams, 2017).

Recientemente, se ha dedicado mucha atención al uso de subproductos provenientes del procesamiento de frutas. Tal uso contribuye a un mejor empleo de los recursos disponibles y propone nuevos ingredientes para la industria alimentaria que contienen fibra dietética (O'Shea, Arendt \& Gallagher, 2012; Lima, Lima, Tavares, Costa \& Pierucci, 2014). El uso de subproductos es de interés debido a que, en algunos se han reportado propiedades funcionales, tecnológicas y nutricionales, así como beneficios para la salud, reducción de la contaminación industrial y los costos (Crizel, Rios, Thys \& Flôres, 2015). La industria alimentaria ha mostrado un interés especial en encontrar usos para los subproductos de la industria de los cítricos. De ahí la necesidad de conocer los componentes físicos y nutritivos de las cáscaras de naranja, subproducto de la extracción de jugo (Olabinjo, Ogunlowo, Ajayi \& Olalusi, 2017).

En México, la producción de cítricos como la naranja, limón, mandarina y toronja genera una considerable derrama económica. En los últimos años, la superficie sembrada y cosechada de cítricos ha mantenido una tendencia creciente de $7.5 \%$. La naranja es el cítrico con la mayor participación de hectáreas cosechadas $(60.5 \%)$, le siguen el limón, la mandarina y la toronja (Solleiro \& Mejía, 2019).

La naranja dulce (Citrus $x$ sinensis) es una de las frutas más comunes en el mundo. Los residuos generados a partir de los frutos son altos: las cáscaras constituyen el principal desperdicio y representan alrededor del 45 al $50 \%$ de la masa total de las frutas. El análisis químico de las cáscaras ha 
mostrado que contienen una cantidad de proteínas de $7.15 \%$ y fibra cruda del $12.79 \%$. Estos subproductos pueden emplearse como ingredientes en alimentos procesados, lo que promovería el procesamiento sostenible de frutos de naranja (Olabinjo et al., 2017).

La composición de los residuos varia en relación con el cítrico del cual provengan y del proceso de manufactura al cual son sometidos. El bagazo de los cítricos, por su alto contenido de agua y su potencial contaminante del ambiente, genera un problema a nivel de las plantas industriales, pero ofrece al mismo tiempo una oportunidad de alimentación. $\mathrm{Su}$ principal aporte nutricional lo constituyen los carbohidratos solubles y estructurales fácilmente fermentables que promueven la formación de ácidos propiónico y acético. Son considerados residuos de alto valor energético con algunas limitaciones para su aprovechamiento en fresco, debido al elevado contenido de agua, mayor al $80 \%$ (Fernández, 2015).

Los subproductos del procesamiento de alimentos son fuentes importantes de aditivos alimentarios como la fibra dietética, antioxidantes, ácidos grasos esenciales, antimicrobianos y minerales. El empleo de estos subproductos en la industria alimentaria agrega valor a la producción, contribuye a la formulación de nuevos productos y disminuye las pérdidas (Márquez-Villacorta \& Pretell-Vásquez, 2018). La incorporación de fibra a los productos consumidos diariamente por la población en general es importante y viable; la adición de subproductos de fibra de naranja a los alimentos es una alternativa interesante debido a que esta fibra contiene un alto valor nutricional y una gran cantidad de antioxidantes (Crizel et al., 2015).

La fibra dietética es un ingrediente funcional en los alimentos, al cual se le ha relacionado con efectos benéficos en la salud. El concepto de fibra incluye a los carbohidratos que no son digeridos ni absorbidos en el tubo digestivo, capaces de llegar sin muchos cambios al colon, donde son fermentados por la microbiota intestinal. Se clasifica en soluble e insoluble, donde cada porción posee propiedades fisiológicas distintas. La fibra se ha empleado frecuentemente para tratar y prevenir diversas patologías gastrointestinales, diabetes, dislipemias, hipertensión arterial y obesidad. Además, su consumo se ha relacionado con la prevención de tumores, en especial del cáncer colorrectal. La Asociación Americana de Dietética establece que, el consumo recomendado de fibra en adultos debe ser de 25 a 30 g/día, o de 10 a 13 g/1000 kcal consumidas. Del mismo modo, la Sociedad Española de Nutrición Comunitaria recomienda una ingesta de fibra dietética superior a 25 g/día (Sánchez, Martín, Palma, López, Bermejo \& Gómez, 2015).

Tras su fermentación colónica y la producción de ácidos grasos de cadena corta, en especial butirato, la fibra facilita el incremento en el flujo sanguíneo local, estimula la proliferación de las criptas, mejora la 
proliferación de los colonocitos y ayuda a la regeneración de la mucosa actuando directamente como antiinflamatorio (Rubio, 2002). Cada tipo de fibra tiene propiedades diferentes en función de su solubilidad en agua, viscosidad, capacidad de ser fermentada o la posibilidad de inducir efectos a nivel sistémico (Gómez, de Cos, \& Iglesias, 2002).

Una fuente importante y potencial de fibra dietética son los subproductos industriales de jugo de naranja porque este material está disponible en grandes cantidades, tiene un bajo costo y contiene compuestos bioactivos asociados (Romero-López, Osorio-Díaz, Bello-Pérez, Tovar \& Bernardino-Nicanor, 2011); estos factores hacen que el uso de subproductos de diversas fuentes sea interesante y viable. Se ha reportado que la fibra de naranja contiene un alto contenido de fibra dietética total y son una fuente de compuestos bioactivos como los fenólicos y los carotenoides (Crizel, Araujo, Rios, Rech \& Flôres, 2014; Crizel et al., 2015).

Las investigaciones de Crizel et al. (2015) muestran el impacto de la incorporación de fibra de naranja en fettuccini al evaluar tres concentraciones: 25,50 y $75 \mathrm{~g} / \mathrm{kg}$. Aunque se obtuvo una pasta con un alto contenido de fibra ( $75 \mathrm{~g} / \mathrm{kg}$ ), solo la formulación de $25 \mathrm{~g} / \mathrm{kg}$ fue similar a la pasta control en relación con los atributos sensoriales y presentó una aceptación superior al 75 $\%$.

Debido a las aplicaciones de la fibra dietética en alimentos y en especial, de la fibra procedente de subproductos de la agroindustria, es importante analizarlas para conocer sus propiedades e integrarlas adecuadamente en la formulación de alimentos con propiedades funcionales. El objetivo de este trabajo fue evaluar la composición fisicoquímica, las propiedades funcionales y la calidad microbiológica de harina de subproductos de naranja dulce, provenientes de la extracción de jugo.

\section{Materiales y Métodos}

Harina. La harina de naranja se obtuvo de subproductos provenientes de expendios de jugos. A los residuos sin jugo se les eliminó el flavedo y las semillas manualmente. Se cortó en cuadros de $0.5 \mathrm{~cm}$ aproximadamente y se secó a $55^{\circ} \mathrm{C}$ en estufa durante 12 h. Posteriormente se molió en molino Ciclotek, se tamizó en malla 80 y se almacenó en recipientes de plástico cerrados (Quijano, 1995).

Análisis fisicoquímicos y funcionales. Los análisis proximales se realizaron empleando métodos de la AOAC (1997): humedad (método 925.09), cuantificación de nitrógeno (método 954.01), extracto etéreo (método 920.39), fibra cruda (método 962.09), cenizas (método 923.03) y extracto libre de nitrógeno, por diferencia del resto de los componentes. Los análisis químicos comprendieron fibra dietética total (Prosky, Asp, Schweizer, DeVries \& Furda, 1998) y fibra dietética soluble e insoluble (AOAC, 1997). 
Los análisis funcionales realizados fueron solubilidad, poder de hinchamiento, absorción de agua (Hernández-Medina, Torruco-Uco, Chel-Guerrero, Betancur-Ancona, 2008) y retención de aceite (Chau, Cheung \& Wong, 1997).

Análisis microbiológicos. La concentración de grupos bacterianos se cuantificó por el método de cultivo en superficie (Corona \& Jiménez, 2004): aerobios mesófilos (agar Métodos Estándar, cat. 211724; BD BioxonTM), coliformes totales (agar Bilis Rojo Violeta, cat. 214300, BD BioxonTM), mohos y levaduras (agar Dextrosa y Papa, cat. 211900, BD BioxonTM) y Salmonella sp. (agar Salmonella-Shigella, cat. 214400, BD BioxonTM). Se incubaron a $37^{\circ} \mathrm{C}$ durante $24-48 \mathrm{~h}$.

\section{Resultados y Discusión}

Composición proximal. El análisis químico permite conocer la composición de los alimentos y agrupar sus componentes en categorías de interés nutricional. El cuadro 1 muestra la composición proximal de la harina de subproductos de naranja Citrus $x$ sinensis, donde el mayor componente corresponde al extracto libre de nitrógeno (ELN) y el menor, al extracto etéreo. Los resultados se comparan con los obtenidos por Haque, Hanif, Nadeem, Mehmood, Ibrar, Iqbal y Jabbar (2015) y Martínez-Fernández, Navarro-Cruz, Vera-López y Avila (2017) en harina de pulpa de cítricos.

Cuadro 1. Composición fisicoquímica de harina de subproductos de naranja.

\begin{tabular}{lccc}
\hline \multicolumn{1}{c}{ Determinación } & $\begin{array}{c}\text { Harina de naranja } \\
(\%)\end{array}$ & Haque et al, 2015 & $\begin{array}{c}\text { Martínez-Fernández } \\
\text { et al, 2017 }\end{array}$ \\
\hline $\mathrm{pH}$ & $4.29 \pm 0.15$ & $3.81 \pm 0.46$ & - \\
Humedad & $8.73 \pm 0.11$ & $8.10 \pm 0.14$ & 3.31 \\
Cenizas & $3.33 \pm 0.74$ & $2.55 \pm 0.04$ & 4.86 \\
Proteína cruda & $5.63 \pm 0.14$ & $7.40 \pm 0.16$ & 5.07 \\
Extracto etéreo & $0.59 \pm 0.08$ & $2.19 \pm 0.08$ & 1.64 \\
Fibra cruda & $11.27 \pm 0.10$ & $7.31 \pm 0.10$ & - \\
Extracto libre de nitrógeno & $79.18 \pm 0.22$ & - & - \\
\hline
\end{tabular}

En esta investigación se obtuvo harina de naranja con una humedad de $8.75 \pm 0.11 \%$, secada a $55^{\circ} \mathrm{C}$. Esta técnica permitió obtener harina de naranja con los valores adecuados de humedad que permiten su almacenamiento a temperatura ambiente. El resultado se encuentra dentro de los parámetros indicados por las normas para harina de trigo, ya que de acuerdo con la norma mexicana NMX-F-007-1982, se recomienda una humedad por debajo de 14.0 $\%$. De igual manera, la norma del Codex Alimentarius CODEX STAN 1521985, recomienda un máximo de $15.5 \%$ de humedad.

La humedad es uno de los factores intrínsecos de los alimentos que permite decidir sobre su recepción, calidad, producción y almacenamiento. La concentración de agua en los alimentos puede afectar su vida útil y las condiciones de almacenamiento; es un factor que puede ser adverso para la 
calidad, principalmente en alimentos secos, como polvos para elaborar bebidas, leches en polvo o recubrimiento de dulces (Carrillo \& Reyes, 2013). Además, un elevado contenido de humedad influye en la velocidad de multiplicación de los microorganismos, provocando su descomposición y por lo tanto la pérdida de la calidad sanitaria (Norma Oficial Mexicana, NOM116-SSA1-1994).

Por otra parte, la concentración de proteínas en la harina de este estudio fue de $5.63 \pm 0.14 \%$. En alimentación humana, la importancia de las proteínas está asociada a su capacidad de aportar aminoácidos para el mantenimiento de la proteína corporal y al incremento de ésta durante el crecimiento; la limitación en el aporte de energía y de proteína a la dieta conduce a un retraso en el crecimiento. En el adulto, la pérdida de proteína corporal se asocia con numerosas alteraciones patológicas y a un aumento en la mortalidad (Martínez \& Martínez, 2006).

Los valores obtenidos en este estudio concuerdan con los analizados en harinas de cítricos realizadas por otros investigadores. Se ha reportado concentraciones de proteínas en naranja de $8.72 \pm 0.36$ (Sharoba, Farrag, \&

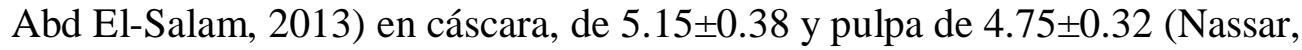
AbdEl-Hamied \& El-Naggar, 2008), así como los reportados por Haque et al, 2015 y Martínez-Fernández et al, 2017, como se muestra en el Cuadro 1.

Al comparar la concentración de proteínas en harina de cítricos con otros subproductos vegetales, se encontró en fibra de manzana una concentración de similar, de $8.03 \pm 0.37$ (Kohajdová, Karovičová, Jurasová, Kukurová, 2011), mientras que, en harina de trigo se han reportado valores de $11.85 \pm 0.40$, en orujo de zanahoria (10.06 \pm 0.18$)$, cáscaras de papa (12.16 \pm $0.48)$ y cáscaras de guisante verde $(13.27 \pm 0.51)$ y harina de piel de papaya Havai (18.18 \pm 0.29) los cuales son mayores que en la harina de cítricos (Sharoba et al., 2013; dos Santos, de Abreu, Freire, Queiroz \& Mendonça, 2014). Sin embargo, en harina de subproductos de cacahuate, las proteínas solamente alcanzan una concentración de $0.46 \%$, muy por debajo de la harina de cítricos (Guerrero-Colín, Trejo-Márquez, Moreno-Lara, Lira-Vargas \& Pascual-Bustamante, 2016).

Las proteínas son uno de los componentes que tecnológicamente son importantes y que determinan la calidad de productos para alimentación humana (de la Vega, 2009). Sin embargo, todos los análisis proximales son de relevancia en la formulación de alimentos. Los resultados obtenidos en cenizas y extracto etéreo concuerdan con los reportados en otros estudios donde se ha analizado la composición proximal de la harina de subproductos cítricos, como los reportados por Haque et al. (2015) y Martínez-Fernández et al. (2017).

Contenido de fibra dietética. La fibra dietética es otro componente importante en los alimentos de origen vegetal. El cuadro 2 muestra los 
resultados obtenidos en esta investigación, alcanzando una concentración cercana al $40 \%$, donde, la fibra dietética soluble e insoluble se encuentran a concentraciones similares. Estos resultados en la concentración de fibra dietética son similares a los publicados por Mosa y Kkalil (2015), así como los de Romero-Lopez et al. (2011), donde se observa una alta concentración de este componente en la harina de subproductos cítricos.

Cuadro 2. Fibra dietética en harina de subproductos de naranja.

\begin{tabular}{cccc}
\hline Determinación & Naranja (\%) & Mosa \& Kkalil, 2015 & $\begin{array}{c}\text { Romero-Lopez et al, } \\
\mathbf{2 0 1 1}\end{array}$ \\
\hline Fibra dietética total & $39.52 \pm 2.08$ & $46.36 \pm 0.03$ & $41.5 \pm 0.0$ \\
Fibra dietética soluble & $20.05 \pm 1.71$ & $19.45 \pm 0.05$ & $18.6 \pm 0.8$ \\
Fibra dietética & $19.02 \pm 1.34$ & $26.91 \pm 0.04$ & $22.9 \pm 0.6$ \\
insoluble & & & \\
FDI/FDS & 0.95 & 1.38 & 1.23 \\
FDI/FDT \% & 48.13 & 58.05 & 55.18 \\
FDS/FDT \% & 50.73 & 41.95 & 44.82 \\
\hline
\end{tabular}

En productos alimenticios, no sólo es importante la concentración de fibra dietética total, sino también la concentración de fibra dietética soluble e insoluble y la relación entre estás. Las relaciones FDI/FDT alrededor del 50 $\%$ es importante, ya que indica una relación equivalente de ambos tipos de fibras. Los investigadores Gorinstein, Zachwieja, Folta, Barton, Piotrowicz, Zemser, Weisz, Trakhtenberg \& Martín-Belloso (2001) sugieren que el mejor efecto proporcionado por la fibra dietética se obtiene en una proporción de 50 a $70 \%$ de fibra insoluble y 30 a $50 \%$ de fibra soluble.

Por otra parte, al comparar el contenido de fibra dietética de la harina de naranja con otras fuentes, se encontró muy por arriba del contenido de frutas como la manzana, donde la cáscara contiene solamente $0.91 \pm 0.09 \%$ de fibra dietética total, aunque con igual contenido de fibra soluble e insoluble (Gorinstein et al, 2001) y, de acuerdo con Dhingra, Michael, Rajput y Patil (2012) en manzana sin pelar, la concentración de fibra dietética total es de 2.0, la insoluble de1.8 y la soluble de $0.2 \mathrm{y}$, en cáscara de papaya, $33.05 \pm 0.70$ (dos Santos et al., 2014). Sin embargo, en residuos de zanahoria se encontró un contenido de fibra dietética total por arriba de la harina de naranja (69.85 $\%$ ), en cáscaras de papa, $73.25 \%$ y en cáscaras de guisante verde $71.3 \%$ (Sharoba et al., 2013).

Aunque en algunos vegetales, la concentración de fibra dietética total es mayor que en la harina de cítricos, la relación entre fibra insoluble y soluble se encuentra en mayor concentración hacia la fibra dietética insoluble, como en el caso de la fibra de zanahoria (insoluble $45.12 \pm 1.08$, soluble $24.73 \pm$ 1.22 ), cáscara de zanahoria (insoluble $53.39 \pm 0.94$, soluble $19.86 \pm 1.47$ ), cáscara de guisante verde (insoluble $51.48 \pm 1.34$, soluble $19.82 \pm 1.36$ ) 
(Sharoba et al., 2013) y cáscara de papaya Havai (insoluble $26.10 \pm 0.56$, soluble $6.95 \pm 0.25$ ), reportados por dos Santos et al. (2014).

La fibra dietética ingerida en la alimentación ejerce un efecto intestinal al retener agua, por lo que las heces tienden a ser más blandas, voluminosas y pesadas. Este incremento del volumen fecal da lugar al aumento del estímulo reflejo que impulsa el peristaltismo con el cual las heces atraviesan el colon más rápidamente, mejorando la evacuación intestinal (Esteva, 2001). La fibra presente en los vegetales facilita el tránsito digestivo, reduce el índice glicémico de los alimentos, mejora la respuesta a la insulina y protege contra enfermedades crónicas degenerativas y la obesidad. Por ello se considera a la fibra como un componente importante de la dieta saludable (Biasoli, Zanuttini, Llopart \& Aimartetti, 2017). Sin embargo, los niveles actuales de ingesta de fibra y alimentos con alto contenido de fibra todavía están muy por debajo de los valores recomendados (Olive \& Komarek, 2017).

En la alimentación humana no solamente es importante la fibra dietética soluble sino también la insoluble ya que la ingestión de cada una de ellas ofrece beneficios a la salud de diferente manera. En general, la fibra soluble retrasa el tiempo de tránsito en el estómago y en el intestino delgado, son rápidamente fermentadas por bacterias del colon y no producen efecto laxante, mientras que las fibras insolubles tienen efecto laxante y no son fermentadas o lo son muy escasamente y contribuyen a la formación del bolo fecal (Gómez et al, 2002).

En trabajos realizados por Ramírez-Camargo, Marulanda \& Orrego (2016) caracterizaron la fibra obtenida de residuos de naranja y reportaron un contenido de fibra dietética total de $35.4 \%$ a $36.9 \%$ en base seca y su potencial uso como espesante, gelificante y texturizante. La fibra obtenida de cítricos tiene una gran ventaja frente a las de cereales, porque aporta una mayor cantidad de fibra dietaría soluble, aproximadamente un $33 \%$ comparado con la fibra del salvado de trigo que solo aporta un $7 \%$. Por esta razón se incluye en productos cárnicos, incrementando el rendimiento en el proceso de cocción, debido a las propiedades funcionales como ligazón de agua y grasa, mejorando la textura en embutidos cocidos y madurados.

Caracterización funcional. Se determinó la capacidad de absorción de agua y aceite de harina de subproductos de naranja, a una temperatura de 30 ${ }^{\circ} \mathrm{C}$; se encontró una mayor capacidad de retención de agua, en comparación con la capacidad de retención de aceite, siendo la relación entre ambos parámetros de 1.45, como se muestra en el cuadro 3 donde, además, se comparan los resultados de la harina de naranja con los obtenidos por otros investigadores en mango, papaya y trigo. Al analizar los resultados, se observa que la cáscara de naranja posee una mayor capacidad de absorción de aceite que la pulpa (Haque et al., 2015; Mosa \& Kkalil, 2015). En relación a otras fuentes de fibra, la pulpa de papaya es una harina con una gran capacidad de 
retención de agua, mientras que la harina de trigo posee muy bajos valores (Varastegani, Zzaman \& Yang, 2015).

Cuadro 3. Propiedades funcionales de harina de naranja y otros subproductos vegetales.

\begin{tabular}{|c|c|c|c|c|}
\hline Fibras & $\begin{array}{c}\text { Capacidad de } \\
\text { absorción de agua }\end{array}$ & $\begin{array}{l}\text { Capacidad de } \\
\text { absorción de } \\
\text { aceite }\end{array}$ & $\begin{array}{c}\text { Relación } \\
\text { agua/aceite }\end{array}$ & Referencias \\
\hline Harina de naranja & $5.72 \pm 0.12$ & $3.95 \pm 0.07$ & 1.45 & \\
\hline $\begin{array}{l}\text { Fibra de pulpa de } \\
\text { naranja }\end{array}$ & $4.71 \pm 0.08$ & $1.91 \pm 0.13$ & 2.47 & Haque et al., 2015 \\
\hline Cáscara de naranja & $5.76 \pm 0.14$ & $7.32 \pm 0.12$ & 0.79 & Mosa \& Kkalil, 2015 \\
\hline Cáscara de mango & $6.08 \pm 0.04$ & $4.02 \pm 0.34$ & 1.51 & Mosa \& Kkalil, 2015 \\
\hline $\begin{array}{l}\text { Harina de pulpa de } \\
\text { papaya }\end{array}$ & $10.55 \pm 0.23$ & $2.47 \pm 0.15$ & 4.27 & $\begin{array}{l}\text { Varastegani, Zzaman } \\
\text { \& Yang, } 2015\end{array}$ \\
\hline Harina de trigo & $1.95 \pm 0.08$ & $1.05 \pm 0.13$ & 1.85 & $\begin{array}{l}\text { Varastegani et al., } \\
2015\end{array}$ \\
\hline
\end{tabular}

Las fibras se diferencian entre sí por su composición y propiedades funcionales, las cuales se determinan, por lo general, in vitro y sirven para obtener una idea de su comportamiento in vivo, ya que en este medio están sometidas a un entorno fisiológico muy complejo y a una serie de mecanismos que pueden modificarlas (Valencia y Román, 2006). Fisiológicamente, la mayor capacidad de absorción de agua contribuye a mejorar el bolo fecal debido a que mejora la evacuación y la retención de aceite contribuye a la excreción de grasas, impidiendo su absorción en el intestino delgado, lo cual contribuye a evitar la acumulación excesiva de grasas en el cuerpo (Gómez et $a l, 2002)$.

Por otra parte, en este estudio se evaluó el efecto de la temperatura sobre la solubilidad, absorción de agua y poder de hinchamiento. Se encontró que el efecto de la temperatura fue mínimo, ya que el comportamiento en estas propiedades fue muy similar en las diferentes temperaturas evaluadas $(60,70$, 80 y $90{ }^{\circ} \mathrm{C}$ ), como se observa en la figura 1 . Sólo la solubilidad se vio afectada por la temperatura, manteniendo una tendencia a incrementar ligeramente con el incremento de la temperatura. 


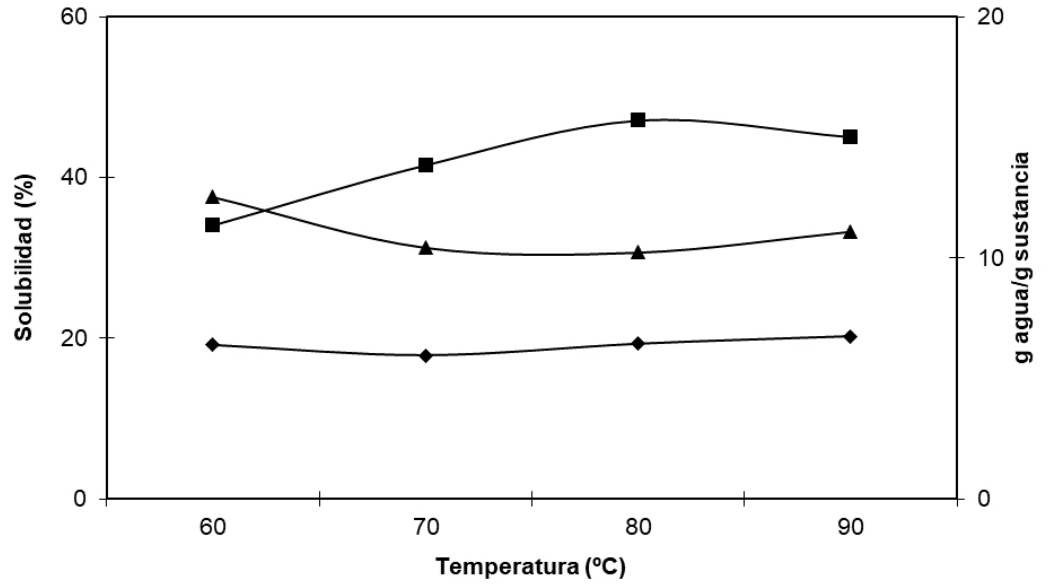

Figura 1. Solubilidad (অ), absorción de agua $(\boldsymbol{\Delta})$ y poder de hinchamiento $(\diamond)$ de harina de naranja.

La estabilidad de la fibra a cambios de temperatura hace posible que pueda emplearse como aditivo en alimentos cocidos, ya que este proceso no altera sus propiedades funcionales, como la absorción de agua, solubilidad o poder de hinchamiento. En este aspecto, la harina de naranja se comporta de manera muy diferente a las harinas amiláceas, ya que las propiedades funcionales de estas últimas se correlacionan en gran medida con el aumento de la temperatura (Contreras-Pérez, De la Torre-Gutiérrez, González-Cortés, \& Jiménez-Vera, 2018). Cuando las fibras son utilizadas en la elaboración de alimentos, las propiedades fisicoquímicas influyen también en las características físicas de éstos, especialmente cuando se usan como agentes gelificantes, espesantes o estabilizantes (Valencia \& Román, 2006).

Las propiedades funcionales de las fibras indican su uso en la formulación de alimentos. En este trabajo se obtuvo un incremento en la capacidad de absorción de agua al analizarla a temperaturas entre $30^{\circ} \mathrm{C}$ y 60 $90{ }^{\circ} \mathrm{C}$. Con el incremento de la temperatura se observó un incremento del doble en esta propiedad funcional. Estos resultados son similares a los reportados por Chaparro, Gil, y Aristizábal (2011), quienes analizaron el efecto de la hidratación y la cocción sobre las características físicas y funcionales en harina de vitabosa (Mucuna deeringiana). Encontraron que la capacidad de absorción de aceite fue superior en la vitabosa sin tratamiento que, en la sometida a hidratación y cocción, mientras que la mayor capacidad de absorción de agua se presentó en la vitabosa sometida a hidratación y cocción.

Se ha demostrado que la capacidad de absorción de agua depende principalmente de las proteínas y de algunos de sus parámetros, como tamaño, forma, factores estéricos y balance hidrofílico-hidrofóbico de los aminoácidos 
en las moléculas, como también de los lípidos y carbohidratos. La cocción de la fibra puede ocasionar una desnaturalización parcial de las proteínas, afectando sus propiedades fisicoquímicas y funcionales. Los cambios conformacionales pueden incrementar aproximadamente un $10 \%$ de la capacidad de absorción de agua de la proteína desnaturalizada (Chaparro et al., 2011).

En panificación, la absorción de agua de una harina es un factor importante ya que de sus propiedades dependerá la calidad del producto, su vida útil y el rendimiento del proceso. Son varios los factores que inciden en este parámetro como el contenido de grasas, proteínas, cenizas, fibras y humedad (Cerda-Mejía, Cerda, Pilamala, Moreno \& Pérez, 2017).

Actualmente, los productores se esfuerzan por crear productos que contengan un factor de valor agregado, como la fibra dietética. Las investigaciones han demostrado que algunos subproductos del procesamiento de las frutas puedan tener un alto valor nutricional. También se ha reportado que pueden ser utilizados como aditivos alimentarios debido a sus propiedades funcionales, como la gelificación y la retención de agua. Las empresas innovadoras buscan continuamente ingredientes económicos, pero de un gran valor agregado (O'Shea et al., 2012).

Caracterización microbiológica. Debido al origen de las sustancias como residuos de la extracción de jugo y a su alta capacidad para ser fermentados, se evaluó la presencia de microorganismos indicadores. En el cuadro 4 se muestran los resultados de la caracterización microbiológica, donde se observa que en la harina los microorganismos presentes en mayor concentración fueron los hongos, seguidos por las levaduras y los mesófilos aerobios, mientras que los coliformes fueron los de menor concentración y Salmonella sp, estuvo ausente.

Cuadro 4. Concentración de grupos microbianos indicadores en harina y pectina de naranja.

\begin{tabular}{lcc}
\hline $\begin{array}{c}\text { Microorganismo } \\
\boldsymbol{s} \text { Log UFC/g) }\end{array}$ & Harina & NOM-147-SSA1-1996 \\
\hline Mesófilos & $4.62 \pm 0.28$ & 5.70 \\
Coliformes & $3.47 \pm 0.21$ & 2.70 \\
Hongos & $5.45 \pm 0.21$ & 2.70 \\
Levaduras & $4.73 \pm 0.51$ & - \\
Salmonella sp. & Negativo & - \\
\hline
\end{tabular}

De acuerdo a la norma oficial mexicana NOM-147-SSA1-1996, sólo el grupo de mesófilos aerobios estuvo dentro de los valores recomendados por dicha norma, mientras que coliformes y hongos, superaron el límite máximo permitido. En el caso de los hongos, su determinación es importante ya que, 
durante el almacenaje, estos microorganismos causan la mayoría de problemas por calentamiento, compactación y deterioro, como en el caso de los granos. Se debe controlar la humedad y temperatura del grano para evitar el desarrollo de hongos (Bolívar, 2007).

\section{Conclusión}

Los análisis fisicoquímicos, funcionales y microbiológicos de la harina de subproductos de naranja (Citrus $x$ sinensis) mostraron que el producto posee la humedad requerida para su almacenamiento, así como la concentración de proteínas adecuadas para su empleo en la formulación de alimentos, siendo el mayor componente el extracto libre de nitrógeno y el menor, al extracto etéreo. Por otra parte, esta harina es una fuente importante de fibra dietética, con un índice recomendado en la relación de la fibra soluble e insoluble para estimular efectos en la salud. A temperatura ambiente, la harina presentó una capacidad de absorción de agua y aceite similar a las reportadas en otros estudios. Sin embargo, la capacidad de absorción de agua se incrementó al doble al incrementar la temperatura, por lo que está fibra puede ser empleada en productos alimentarios sometidos a cocción. En relación a la calidad microbiológica, los mesófilos se encuentran dentro de los valores máximos permitidos, siendo importante disminuir los valores de coliformes y hongos, ya que se encuentran fuera de la norma para harina de trigo. La harina de subproductos de naranja puede ser empleada como aditivo en la formulación de alimentos, capaz de proporcionar valor agregado a la cadena de cítricos al ser una fuente importante de nutrientes para la alimentación humana.

\section{References:}

1. AOAC. (1997). Association of Official Analytical Chemist. Official Methods of Analysis. (17ta. ed.). Washington, D. C., USA.

2. Biasoli, M., Zanuttini, L., Llopart, E. \& Aimartetti, N. (2017). Postre a base de quinua para niños. Actualización en Nutrición. 18(1):27-36.

3. Bolívar, M. (2007). Manejo de granos en almacenamiento, causas de deterioro y prevención. Arch. Latinoam. Prod. Anim. 15(Supl. 1):180184.

4. Carrillo, M. \& Reyes, A. (2013). Vida útil de los alimentos. Revista Iberoamericana de las Ciencias Biológicas y Agropecuarias. 2(3):1-32.

5. Cerda-Mejía, L., Cerda, V., Pilamala, A., Moreno, C. \& Pérez, A. (2017). Proteína de harinas de maíz, cebada, quinua, trigo nacional y papa: características y funcionalidad como sustitutos de la proteína de harina de trigo importado en la producción de pan y fideos. Revista Amazónica Ciencia y Tecnología. 6(3):201-216. 
6. Chaparro, S., Gil, J. \& Aristizábal, I. (2011). Efecto de la hidratación y la cocción en las propiedades físicas y funcionales de la harina de vitabosa (Mucuna deeringiana). VITAE, Revista de la Facultad de Química Farmacéutica. 18(2):133-143.

7. Chau, C., Cheung, P. \& Wong, Y. (1997). Functional properties of protein concentrates from three chinese indigenous legume seeds. J. Agric. Food Chem. 45:2500-2503.

8. Codex Alimentarius, CODEX STAN 152-1985. Norma del Codex para la harina de trigo. CODEX STAN 152-1985. Adoptado 1985. Revisión 1995.

9. Contreras-Pérez, R., De la Torre-Gutiérrez, L., González-Cortés, N. \& Jiménez-Vera, R. (2018). Caracterización funcional de almidones de plátano cuadrado (Musa balbisiana Colla). European Scientific Journal. 14(30):82-97.

10. Corona, A. \& Jiménez, R. (2004). Comparación de dos métodos de siembra para el recuento de microorganismos en muestras con alta concentración microbiana. Revista de la Facultad de Ingeniería Química. (40):3-7.

11. Crizel, T., Araujo, R., Rios, A., Rech, R. \& Flôres, R. (2014). Orange fiber as a novel fat replacer in lemon ice cream. Food Sci. Technol, Campinas. 34(2):332-340.

12. Crizel, T., Rios, A., Thys, R. y Flôres, S. (2015). Effects of orange byproduct fiber incorporation on the functional and technological properties of pasta. Food Sci. Technol, Campinas. 35(3):546-551.

13. Cury, K., Aguas, Y., Martinez, A., Olivero, R. y Chams, L. (2017). Residuos agroindustriales su impacto, manejo y aprovechamiento. Rev Colombiana Cienc Anim. 9(Supl):122-132.

14. de la Vega, G. (2009). Proteínas de la harina de trigo: clasificación y propiedades funcionales. Temas de Ciencia y Tecnología. 13(38):2732.

15. Dhingra, D., Michael, M., Rajput, H. \& Patil, R. (2012). Dietary fibre in foods: a review. J Food Sci Technol. 49(3):255-266.

16. dos Santos, C., de Abreu, C., Freire, J., Queiroz, E. \& Mendonça, M. (2014). Chemical characterization of the flour of peel and seed from two papaya cultivars. Food Sci. Technol, Campinas. 34(2):353-357.

17. Esteva, E. (2001). Trastornos de la función intestinal. Diarrea y estreñimiento. OFFARM. 20(3):80-90.

18. Fernández, A. (2015). Subproductos de los cítricos. Sitio Argentino de Producción Animal. Disponible en: http://www.produccionanimal.com.ar/tablas_composicion_alimentos/131-

Subproductos_citricos.pdf 
19. Guerrero-Colín, J., Trejo-Márquez, M., Moreno-Lara, J., Lira-Vargas, A. y Pascual-Bustamante, S. (2016). Extracción de fibra de los desechos agroindustriales de cacahuate, para su aplicación en el desarrollo de alimentos. Investigación y Desarrollo en Ciencia y Tecnología de Alimentos. 1(2):806-812.

20. Gómez, C., de Cos, B. \& Iglesias, R. (2002). Fibra y Nutrición Enteral. Nutr Hosp. 17(Sup. 2):30-40.

21. Gorinstein S., Zachwieja Z., Folta M., Barton H., Piotrowicz J., Zemser M., Weisz M., Trakhtenberg S. \& Martín-Belloso O. (2001). Comparative contents of dietary fiber, total phenolics, and minerals in persimmons and apples. J. Agric. Food Chem. 49:952-957.

22. Haque, E., Hanif, M., Nadeem, M., Mehmood, A., Ibrar, M., Iqbal, Z. \& Jabbar, S. (2015). Physicochemical and rheological study of orange pulp fortified cookies. Sci Lett. 3(2):64-67.

23. Hernández-Medina, M., Torruco-Uco, J., Chel-Guerrero, L. \& Betancur-Ancona, D. (2008). Caracterización fisicoquímica de almidones de tubérculos cultivados en Yucatán, México. Ciênc. Tecnol. Aliment., Campinas. 28(3):718-726.

24. Kohajdová, Z., Karovičová, J., Jurasová, M. \& Kukurová, K. (2011). Effect of the addition of commercial apple fibre powder on the baking and sensory properties of cookies. Acta Chimica Slovaca. 4(2):88-97.

25. Lima, B., Lima, F., Tavares, M., Costa, A. \& Pierucci, A. (2014). Determination of the centesimal composition and characterization of flours from fruit seeds. Food Chemistry. 151(2014):293-299.

26. Márquez-Villacorta, L. \& Pretell-Vásquez, C. (2018). Evaluación de características de calidad en barras de cereales con alto contenido de fibra y proteína. Biotecnología en el Sector Agropecuario y Agroindustrial. 16(2):67-78.

27. Martínez-Fernández, E., Navarro-Cruz, A., Vera-López, O. \& Avila, R. (2017). Caracterización Fisicoquímica de desechos de naranja (Citrus Sinensis) y lechuga (Lactuca Sativa). Revista de Energía Química y Física. 4(10):49-56.

28. Martínez, O. \& Martínez, E. (2006). Proteínas y péptidos en nutrición enteral. Nutr. Hosp. 21(Supl. 2):1-14.

29. Mosa, Z. \& Kkalil, A. (2015). Comparative study between the effects of mango and orange peels preparations on the total dietary fiber. IOSR Journal of Environmental Science, Toxicology and Food Technology. 9 (11):129-136.

30. Nassar, A., AbdEl-Hamied, A. \& El-Naggar, E. (2008). Effect of citrus by-products flour incorporation on chemical, rheological and organolepic characteristics of biscuits. World Journal of Agricultural Sciences. 4(5):612-616. 
31. Norma mexicana NMX-F-007-1982. Alimento para humanos. Harina de trigo. Foods for humans. Wheat flour. Normas mexicanas. Dirección General de Normas.

32. Norma Oficial Mexicana NOM-116-SSA1-1994, bienes y servicios. Determinación de humedad en alimentos por tratamiento térmico. Método por arena o gasa. Dirección General de Normas.

33. Olabinjo, O., Ogunlowo, A., Ajayi, O. y Olalusi, A. (2017). Analysis of physical and chemical composition of sweet orange (Citrus sinensis) peels. International Journal of Environment, Agriculture and Biotechnology. 2(4):2201-2206.

34. Olive, Y. \& Komarek, A. (2017). Dietary fibre basics: Health, nutrition, analysis, and applications. Food Quality and Safety. 1(1):4759.

35. O'Shea, N., Arendt, E. \& Gallagher, E. (2012). Dietary fibre and phytochemical characteristics of fruit and vegetable by-products and their recent applications as novel ingredients in food products. Innovative Food Science and Emerging Technologies. 16(2012):1-10.

36. Prosky, L., Asp N., Schweizer T., DeVries J. \& Furda I. (1998). Determination of insoluble, soluble, and total dietary fiber in foods and food products: interlaboratory study. Journal of the Association of Official Analytical Chemists. 71:1017-1023.

37. Quijano, E. (1995). Extracción de pectina a partir de residuos de la extracción de jugo de naranja. Tesis de la Facultad de Química de la UADY. México.

38. Ramírez-Camargo, E., Marulanda, A. \& Orrego, J. (2016). Desarrollo de una mezcla de fibras y almidones como reemplazante de grasa para productos de pasta fina tipo salchicha. Información Tecnológica. 27(1):41-52.

39. Romero-Lopez, M., Osorio-Diaz, P., Bello-Perez, L., Tovar, J. \& Bernardino-Nicanor, A. (2011). Fiber concentrate from orange (Citrus sinensis L.) bagasse: characterization and application as bakery product ingredient. Int. J. Mol. Sci. 12:2174-2186.

40. Rubio, M. (2002). Implicaciones de la fibra en distintas patologías. Nutr Hosp. 17(Sup. 2):17-29.

41. Sánchez, R., Martín, M., Palma, S., López, B., Bermejo, L. \& Gómez, C. (2015). Indicaciones de diferentes tipos de fibra en distintas patologías. Nutr Hosp. 31(6):2372-2383.

42. Sharoba, A., Farrag, M. \& Abd El-Salam, A. (2013). Utilization of some fruits and vegetables waste as a source of dietary fiber and its effect on the cake making and its quality attributes. Journal of Agroalimentary Processes and Technologies. 19(4):429-444. 
43. Solleiro, J. \& Mejía, O. (2019). Producción de cítricos e innovación: una oportunidad para México. TecnoAgro. 132. Disponible en: https://tecnoagro.com.mx/no.-132/produccion-de-citricos-einnovacion-una-oportunidad-para-mexico

44. Valencia, F. \& Román, M. (2006). Caracterización fisicoquímica y funcional de tres concentrados comerciales de fibra dietaria. VITAE, Revista de la Facultad de Química Farmacéutica. 13(2):54-60.

45. Varastegani, B., Zzaman, W. \& Yang, T. (2015). Investigation on physicochemical and sensory evaluation of cookies substituted with papaya pulp flour. Journal of Food Quality. 38(2015):175-183. 